
ilitary Technical College
obry Elkobbah,
airo, Egypt

\title{
DESIGN, ANALYSIS AND REALIZATION IN MICROSTRIP FORM OF THE DOWNCONVERSION AND IF AMPLIFICATION PARTS OF A MICROWAVE RECEIVER
}

\author{
Esmat* A. A., Mohammed** A. N. and Ayman*** G. S.
}

\begin{abstract}
By the aid of the Microwave Design System (MDS), software package, the design and analysis of the downconversion and IF amplification parts of a microwave superhytrodyne receiver, operating in the S-band, and the implementation of the designed subsystem in the microstrip form, is presented.. All individual circuits constituting this subsystem are fabricated separately and their characteristics are measured to test their performances and then the integration of these circuits is fabricated again on a single substrate and its characteristics are also tested and measured.
\end{abstract}

\section{KEY WORDS}

CAD, S-band, Microstrip, Receiver and Subsystem

\section{INTRODUCTION}

In the last few years, a successful progress has been achieved in the field of using CAD programs in the design and analysis of microwave circuits, subsystems and systems. So, the main objective of this paper is the design and analysis of the downconversion and IF amplification parts of a microwave superhytrodyne receiver operating in the S-band, and the implementation of the designed subsystem in the microstrip form, by the aid of one of the most modern and powerful CAD packages, known by HP Rf \& Microwave Design System (MDS). The proposed subsystem is designed to receive an RF signal at $3.2 \mathrm{GHz}$ to downconvert it into an IF signal of 400 $\mathrm{MHz}$ by means of a single-balanced diode mixer, using an external LO of $3.6 \mathrm{GHz}$, then an IF amplifier circuit is used to compensate for the mixer conversion loss and to provide the $400 \mathrm{MHz}$ IF signal with the required gain.

The proposed subsystem is designed to operate over a large dynamic range, so the first stage is an RF bandpass filter representing a selective circuit to pass certain band of frequencies centered at the frequency of operation $(3.2 \mathrm{GHz})$ [1].

*Professor, Det. Of Microstrip Circuits, Electronics Research Institute, Guiza, Egypt.

${ }^{* *}$ Associate Professor, Det. Of circuits \& systems, M.T.C, Cairo, Egypt.

${ }^{* * *}$ MSc., Det. Of Electronic Engineering , M.T.C, Cairo, Egypt. 
All circuits contained in this subsystem (RF filter, mixer, IF filter and IF amplifier) are designed by the aid of synthesis formulae corresponding to each, and all these synthesis are programmed in $\mathrm{C}++$ language easy to run on any personal computer. The RF \& Microwave Design System (MDS) software package is then used to simulate the performances of the designed separate circuits, by applying the appropriate type of simulation for each circuit. If the given specifications are not satisfied, then one of the optimization techniques, available in the used package, is used to reach the optimum response desired. These circuits are connected to each other representing the proposed subsystem whose performance are simulated and optimized to meet the given specifications. Finally, these circuits are fabricated individually, their characteristics are measured, and then the integrated on a single substrate representing the proposed subsystem, whose characteristics are tested and measured.

\section{DESIGN AND SIMULATION OF THE SEPARATE CIRCUITS}

As stated above, the proposed subsystem consists of four circuits namely, RF bandpass filter, single-balanced diode mixer, IF bandpass filter and IF amplifier. We must note that the mixer circuit requires in its operation a hybrid-coupler to couple both RF \& LO signals to the two diodes of the mixer. The used hybrid here is 3-dB branch-line hybrid (B.L.H) which will be designed and simulated separately similar to other circuits, and this will be done as follows:-

\section{II.1 RF Bandpass Filter}

This filter is a parallel-coupled resonators chebyshev bandpass filter with certain modification in its structure[2] as shown in Fig. 1 .

Performing an S-parameters simulation to this filter circuit, the obtained results are shown in Fig.2. Comparing the performances of the modified filter structure with the traditional parallel-coupled resonators filter, which is also designed and simulated, we found that the modified version yields many benefits over the traditional one such as:-

- The input and output lines are made collinear.

- The new filter takes less space because it can be fitted in quite a narrow channel

- The upper stopband rejection is improved by about $15 \mathrm{~dB}$, which is the major advantage of the modified structure because it greatly improves the image rejection capability of the receiver (fimage $=4 \mathrm{GHz}$ ).

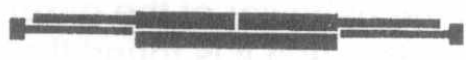

Fig.1. RF bandpass filter

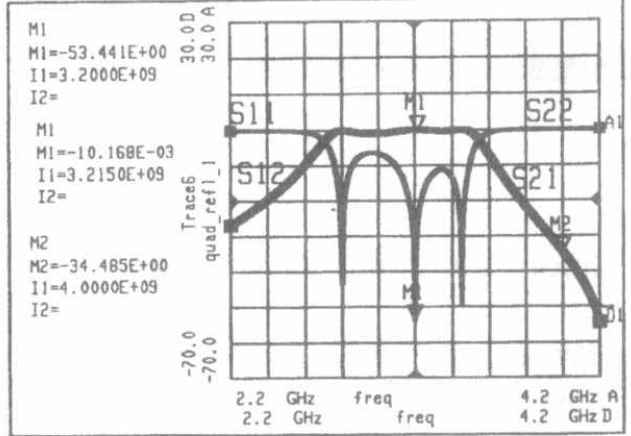

Fig.2. S-parameters simulation results of the RF filter 
Proceedings of the $\mathbf{2}^{\text {nd }}$ ICEENG Conference, 23-25 Nov. 1999

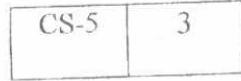

\section{II.2 3-dB Branch-Line Hybrid}

It is required to design a wide bandwidth B.L.H( $>22 \%)$ to provide an accrate equal power division at the two frequencies of interest ( $f_{R F}=3.2 \mathrm{GHz}, f_{L O}=3.6 \mathrm{GHz}$ ). The bandwidth of the single-section B.L.H is as low as it can not be used to perform this task $(11 \%$ at most), and so, we use a double-section B.L.H [3], that is shown in Fig.3. Performing an S-parameters simulation to this hybrid circuit, the results shown in Fig.4 are obtained, from which we can see that it can operate efficiently over a large bandwidth, reaches about $32 \%$, providing an accurate equal power division at the two frequencies of interest.

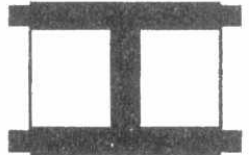

Fig.3. Double-section B.L.H

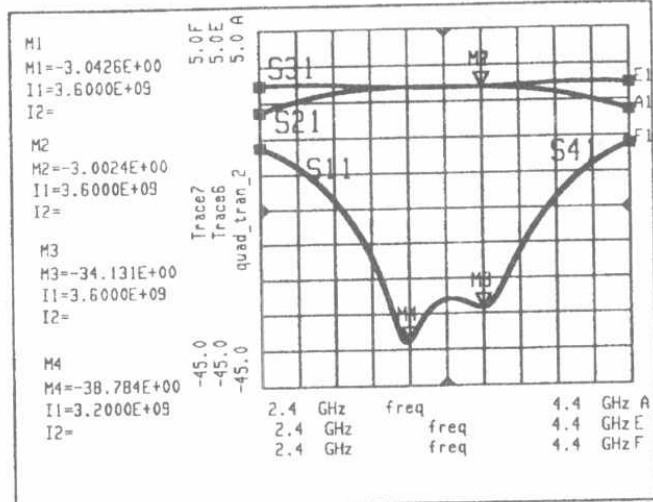

Fig.4. S-parameters simulation results of the B.L.H

\section{II.3 Single-balanced mixer}

The mixer circuit is a single-balanced diode mixer consisting of a pair of SM silicon beam lead schottky diodes. The RF-impedance matching is achieved using singlestub matching networks[4]-[6], while the IF matching circuit is a single $\lambda / 4$ transformer, as shown in Fig.5.Performing a harmonic balance simulation to the obtained mixer circuit with RF input power of $-10 \mathrm{dBm}$ and LO power of $12 \mathrm{dBm}$, the output spectrum at the IF port is shown in Fig.6, from which we can see that the mixer conversion loss reaches about $6.5 \mathrm{~dB}$ which can be practically accepted.

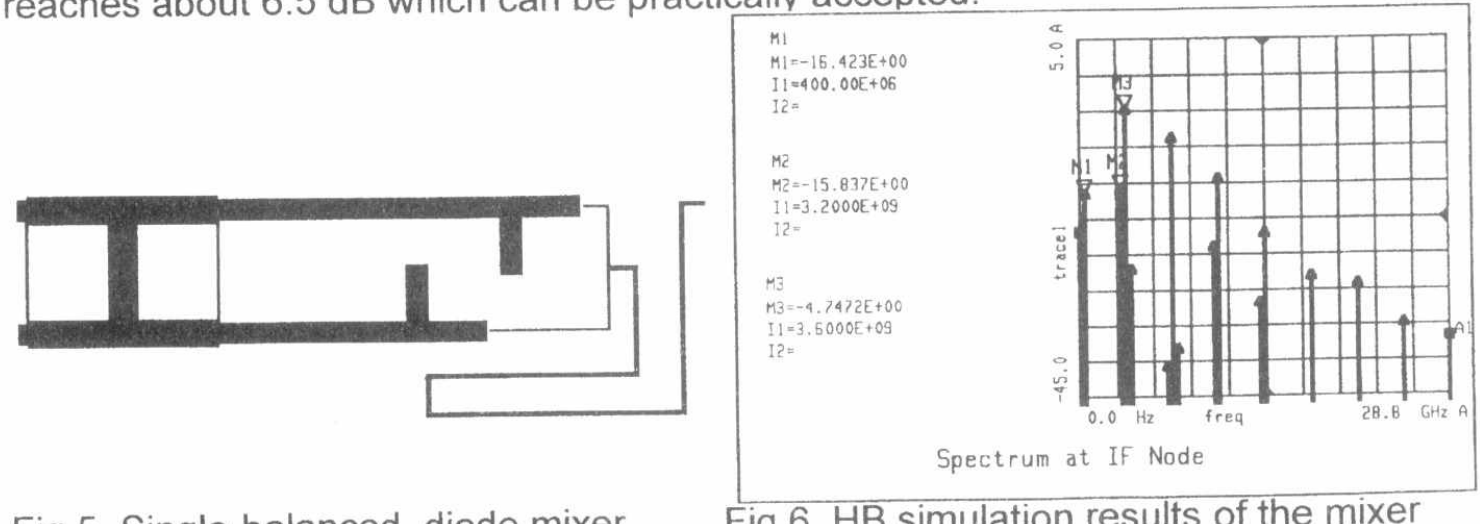

Fig.5. Single-balanced diode mixer

Fig.6. HB simulation results of the mixer

A large sweep is made for the input RF power to predict the behavior of the mixer when different levels of RF signals are detected by the system and it is found that the conversion loss is approximately constant over a wide range of RF power, starting to increase at high levels of RF power $(>6 \mathrm{dBm})$ due to the diode current limitations making it to saturate at these levels. Also, the LO power is swept over a suitable range to check the stability of the mixer against variations in this parameter, and it is found 
Proceedings of the $\mathbf{2}^{\text {nd }}$ ICEENG Conference, $23-25$ Nov. 1999

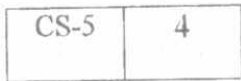

that the conversion loss decreases with increasing LO power till saturation is reached due to the same reason.

\section{II.4 IF bandpass filter}

This circuit is used to filter out all mixing products to pass only the $400 \mathrm{MHz}$ IF signal. It is designed using the modern network synthesis[7], and the circuit shown in Fig.7 is obtained. Obviously, the frequency of operation of this circuit is as low as it can not be realized in the microstrip form, and so, it is realized in the lumped elements form (using chip inductors and capacitors) but, unfortunately, the values of chip inductors and capacitors obtained from filter design $(54.65 \mathrm{nH}, 7.72 \mathrm{nH}, 3 \mathrm{pf}$ ) are not available in the standard international kits of chip elements, accordingly, this filter can not be realized practically. In this case, we have to use the values of inductors and capacitors available in practice, and as close as possible to these obtained from filter design (56 $\mathrm{nH}, 8.2 \mathrm{nH}, 5 \mathrm{pf}$ ) which results in some deviation from the given specifications as shown in Fig.8.
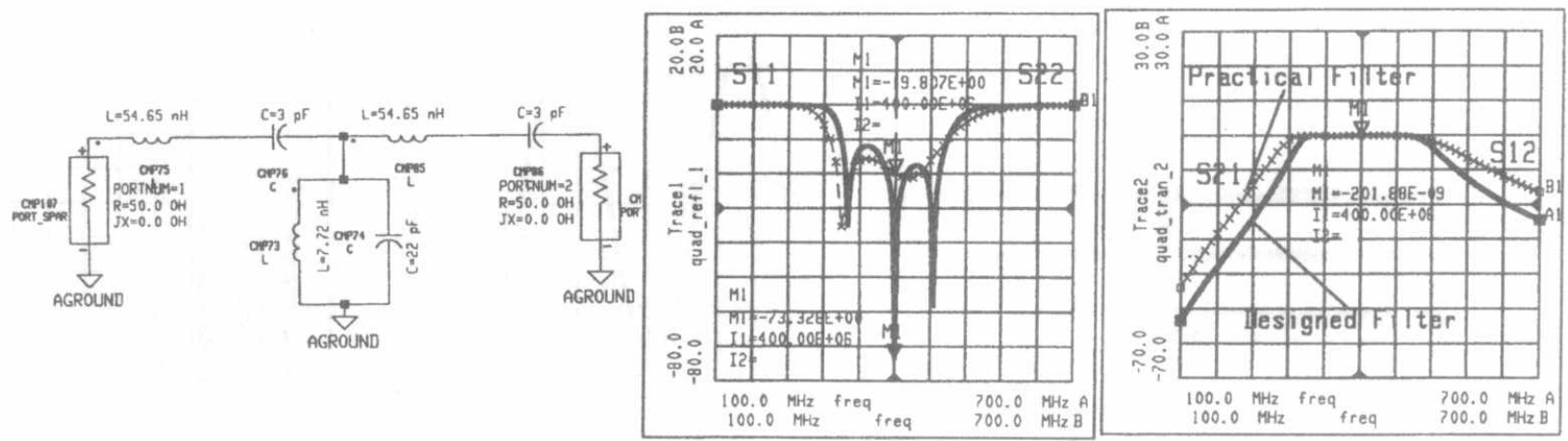

Fig.7. IF bandpass filter

Fig.8. S-parameters simulation results of the if filter

\section{II.5 IF amplifier}

It is a low noise BJT amplifier designed using a linear model for the used transistor whose S-parameters are measured by the network analyzer and transferred directly to the used package to construct this linear model. Also, this circuit is realized in the lumped elements form as shown in Fig.9. The same problem in the chip inductors and capacitors values, discussed in the previous circuit, exists now and Fig.10 shows the deviation from the given specifications in the amplifier circuit due to this problem.
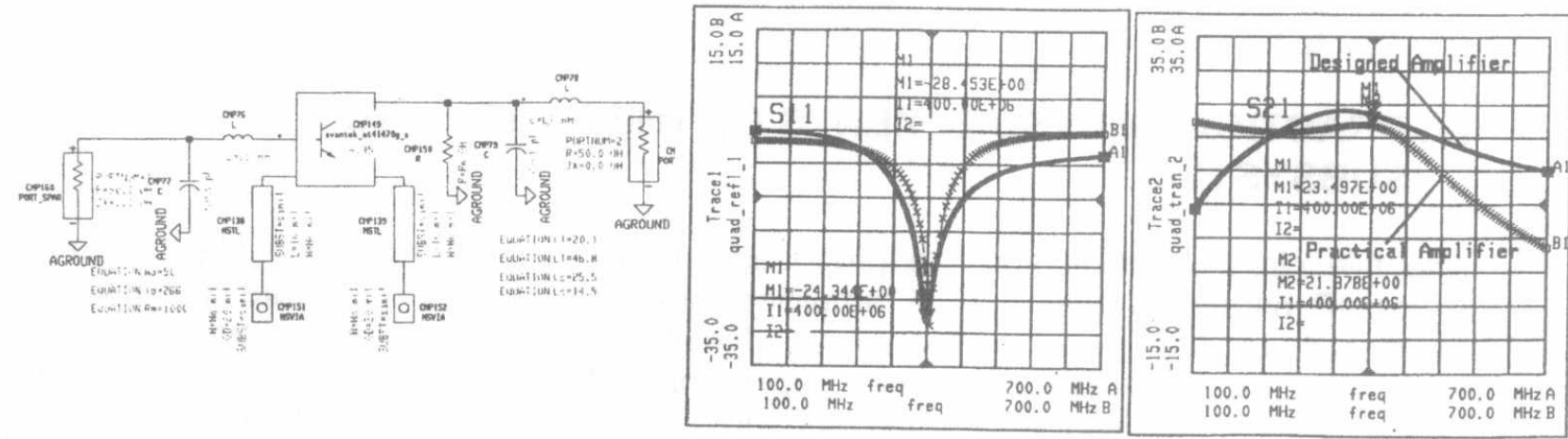

Fig.9. IF amplifier

Fig.10. S-parameters simulation results of the if amplifier. 
Proceedings of the $2^{\text {nd }}$ ICEENG Conference, 23-25 Nov. 1999

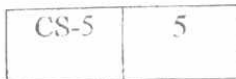

\section{SIMULATING THE PERFORMANCE OF THE DESIGNED SUBSYSTEM}

Now, after the designed separate circuits are optimized for maximum performance, as possible as we could, they are connected to each other according to the manner shown in Fig. 11 (scaled 50\%).

A HB simulation is performed to this subsystem with RF input power of $-10 \mathrm{dBm}$ and LO power of $12 \mathrm{dBm}$. The simulation results are shown in Fig. 12 from which we can see that it provides, at the IF-port, a $400 \mathrm{MHz}$ pure sinusoidal signal with total harmonic distortion $70 \times 10^{-6}$ and total conversion gain of the system reaches $14 \mathrm{~dB}$. Also, we can note that the LO \& RF signals have weak levels at the IF-port (-79 dBm, $87 \mathrm{dBm}$ ) which means an excellent isolation between the LO, RF ports and the IFport. Also, one of the strongest capabilities of this subsystem is the perfect suppression of both image and IF frequencies, if entered at the RF-port. We found that the output IF-signal, resulted in the image and IF frequencies has a negligible power $-43 \mathrm{dBm},-51 \mathrm{dBm}$, respectively.

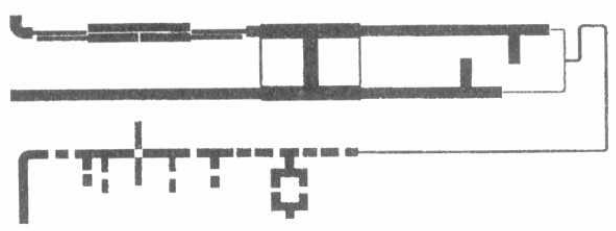

Fig.11. Proposed subsystem
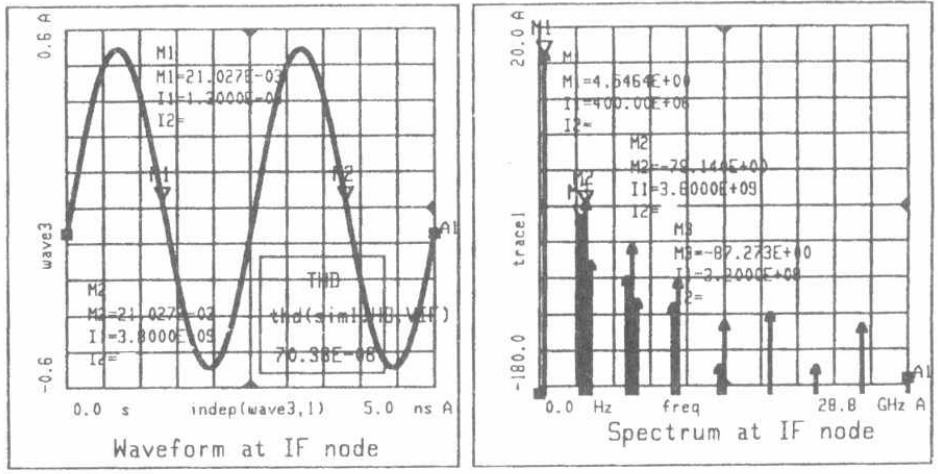

Now the input RF power is swept over a wide range to predict the dynamic range of the system over which the input signals can be processed properly and we get the following information:-

-The minimum detectable signal is approximately $-70 \mathrm{dBm}$, which can be considered as the receiver sensitivity.

-The total conversion gain of the system decreases by $1 \mathrm{~dB}$ at IF output power of 15 $\mathrm{dBm}$ corresponding to RF input of $2 \mathrm{dBm}$ which can be considered as the one- $\mathrm{dB}$ compression point of the system.

-The dynamic range of the system is calculated, as the difference between these two values, to be about $72 \mathrm{~dB}$.

\section{PRACTICAL MEASUREMENTS OF THE FABRICATED SUBSYSTEM}

The five circuits constituting the proposed subsystem ( RF bandpass filter , B.L.H, mixer, IF bandpass filter and IF amplifier) are fabricated as separate circuits and measured to test their performances. Finally, these circuits are integrated and fabricated on a single substrate representing the proposed subsystem whose characteristics are also tested and measured. The S-parameters of all linear circuits, contained in our subsystem, are measured by the network analyzer and the measured 
data are transferred to the MDS to be compared with the simulation results as shown in Fig.13-Fig.16 for the RF -BPF, B.L.H, IF-BPF and IF amplifier, respectively.
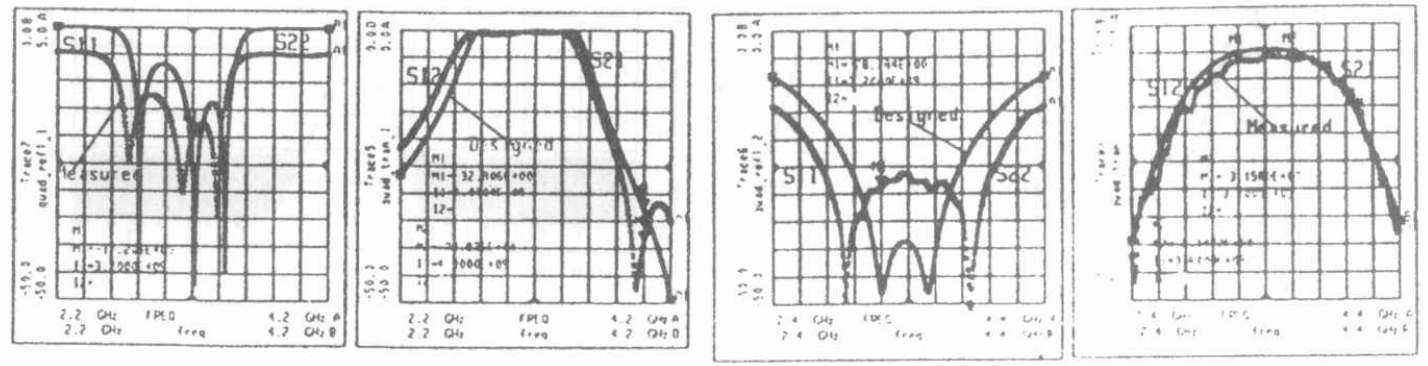

Fig.13. Measured Data of the RF BPF

Fig.14. Measured Data of the B.L.H
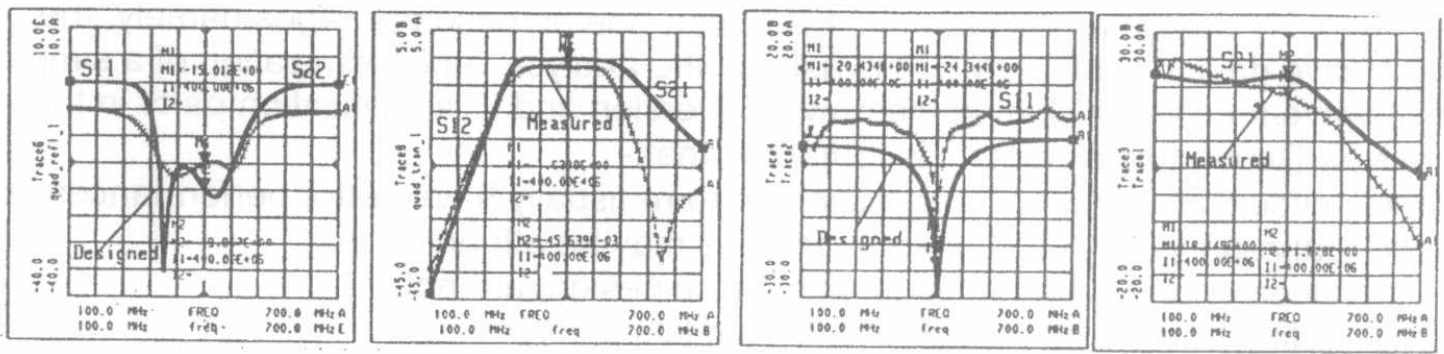

Fig.15. Measured Data of the IF BPF

Fig.16. Measured Data of the IF Amplifier

From these figures we can see that the fabricated linear circuits satisfy most of the design specifications with some deviations which may be due to the fabrication errors or the manufacture tolerances in the chip elements.

The mixer conversion loss is measured using two synthesized sweepers representing the input RF and LO signals and a spectrum analyzer to display the output IF signal spectra. It is found that the mixer circuit has a conversion loss reaches $-8 \mathrm{~dB}$ which is nearly constant over an IF frequency range from $350 \mathrm{MHz}$ up to $750 \mathrm{MHz}$ and RF input power from $-70 \mathrm{dBm}$ up to $5 \mathrm{dBm}$.

Now the integration of these circuits on a single substrate, according to the arrangement proposed to minimize the total size of the subsystem, Fig.11, is measured to evaluate its performances and to check its proper operation. The fabricated subsystem can be considered as a nonlinear circuit whose characteristics can be measured using the same setup used for mixer measurements, after feeding the BJT of the IF amplifier with the required dc bias through an external bias circuit. Doing this, and displaying the output signal on the spectrum analyzer and once again on a digitizing oscilloscope, the results shown in Fig.17 are obtained. From this figure we can conclude that our subsystem provides, at the IF-port, a $400 \mathrm{MHz}$ IF-signal with total conversion gain of the system $9 \mathrm{~dB}$. Also, it is found that the RF, LO frequencies have week harmonics at the IF-port meaning a good isolation between this port and 
the LO, RF ports and decreased total harmonic distortion and this is obvious from the sinusoidal signal displayed on the digitizing oscilloscope.
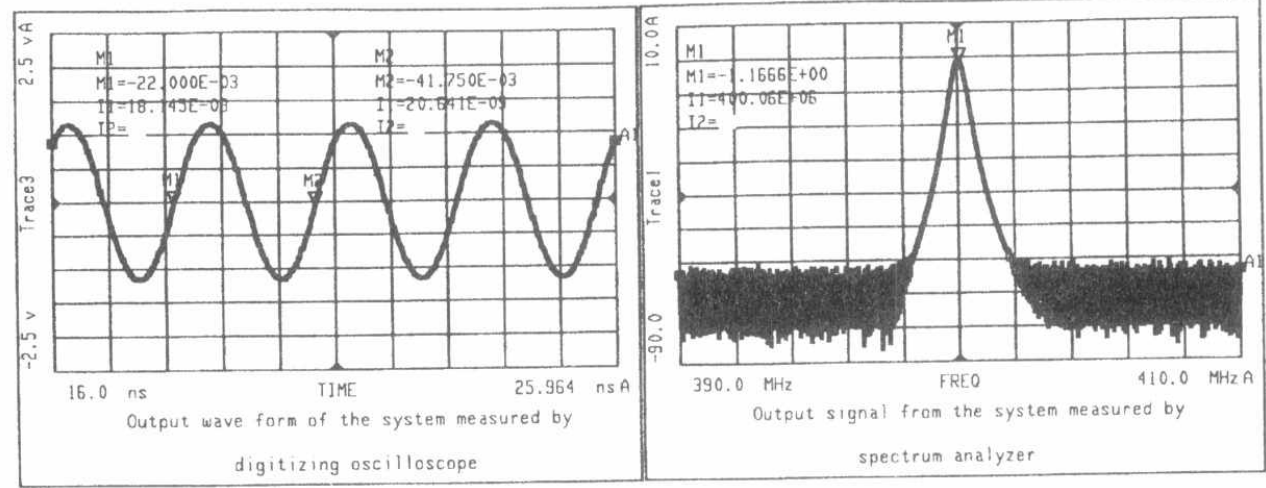

Fig. 17. Measured Data of the Fabricated Subsystem

\section{CONCLUSION}

The CAD software packages are valuable tools in all phases of microwave circuits and systems design procedures. In this paper, one of these packages namely, the microwave design system (MDS) is used in the analysis and optimization of a simple microwave receiver, including the downconversion and IF amplification parts in this receiver. During this process it is remarked that:-

(1) All circuits realized in the microstrip form usually have better performances than those realized by lumped elements, and this may be attributed to:-

- The values of these elements obtained from design, are not always found in the international standard kits.

- The undetectable manufacture tolerances always found in these elements.

(2) The most effective way to increase the receiver sensitivity is to add an RF amplifier in front of the mixer which often decreases the dynamic range of the receiver.

Therefore, a compromise between sensitivity and dynamic range must be carefully evaluated during the receiver design.

\section{References}

[1] J. B. Y Tsui, "Microwave receivers with electronic warfare applications", Centerville, Ohio, March (1986).

[2] C.Y. Chang, T. Itoh, "A modified parallel-coupled filter structure that improves the upper stopband rejection and response symmetry", IEEE Trans. Microwave Theory Tech., vol. MTT-39, No-2, p310-313, February, (1991).

[3] S. Kumar, C. Tannous, T. Danshin, "A multisection broadband impedance transforming branch-line hybrid", IEEE Trans. Microwave Theory Tech., vol. MTT43, No. 11, p2517-2522, November (1995).

[4] I. J. Bahl, "Microwave solid state circuit design", John Wiley and Sons, Inc., U.S.A, (1988).

[5] S. A. Maas, "Microwave mixers" Artech House,Inc., (1993).

[6] Hewlett-Packard, "Mixer and detector diodes" HP Application note 80800,(1993).

[7] W. Max, F. Medley, "Microwave \& RF circuits analysis, synthesis and design", Artech House, 1st edition,(1992). 